\title{
Route Design Model of Multiple Feeder Bus Service Based on Existing Bus Lines
}

\author{
Meina Zheng $\mathbb{D}^{1,2,3}$ Runxuan Zhou $\mathbb{D}^{1,2,3}$ Shanshan Liu $\mathbb{D}^{1,2,3}$ Feng Liu $\mathbb{D}^{4}{ }^{4}$ \\ and Xiucheng Guo $\mathbb{D}^{1,2,3}$ \\ ${ }^{1}$ Jiangsu Key Laboratory of Urban ITS, Southeast University, Nanjing 211189, China \\ ${ }^{2}$ Jiangsu Province Collaborative Innovation Center of Modern Urban Traffic Technologies, Southeast University, \\ Nanjing 211189, China \\ ${ }^{3}$ School of Transportation, Southeast University, Nanjing 211189, China \\ ${ }^{4}$ School of Economics and Management, Southeast University, Nanjing 211189, China \\ Correspondence should be addressed to Xiucheng Guo; seuguo@163.com
}

Received 25 May 2020; Revised 19 November 2020; Accepted 3 December 2020; Published 12 December 2020

Academic Editor: Kun An

Copyright (c) 2020 Meina Zheng et al. This is an open access article distributed under the Creative Commons Attribution License, which permits unrestricted use, distribution, and reproduction in any medium, provided the original work is properly cited.

\begin{abstract}
The feeder bus, as an important component of urban public transportation systems, is capable of filling the existing service gap left by urban rail transit to satisfy feeder demand. Two challenges to taking advantage of the feeder bus are identified. The first challenge is to identify the service gap, and the second one is to strengthen the coordination of multilevel urban traffic modes. Given these two challenges, this paper established a route design model that introduced a feeder bus demand coefficient to quantify feeder demand for each bus stop. To assist the design of feeder bus routes, an optimization approach based on the tabu search algorithm was proposed. Considering the sizeable combinational optimization problem, the algorithm decomposed this problem into three phases: feeder area partition, candidate stop selection, and route design problem. In the first phase, the entire area was partitioned into several subareas and the specific boundary nodes were moved into an adjacent subarea. Then, the stops with the minimum overall travel cost from every subarea were chosen as candidate ones under the limit of operating costs. The last step was to find the shortest circular route to visit each candidate stop and determine the operating direction. The proposed optimization had been implemented in a downtown area of Suzhou, China, in order to verify its effectiveness through the performance in terms of the quality of optimal solutions and computing efficiency. In addition, a possibility of simplifying a multiple circular route design problem to a single route design problem utilizing spatial clustering is provided to enhance the computing efficiency in a large-scale network.
\end{abstract}

\section{Introduction}

Developing public transportation is a crucial way to satisfy travel demand, especially in urban areas. Passengers tend to travel by highly reliable transit service. Urban rail transit can provide rapid, reliable, and safe service for passengers. Traveling through metro mass transit is $20 \%$ cheaper in terms of price [1]. However, many passengers do not live or work within walking distance of existing urban rail stations. To complete their trips, one or more transfers between buses and urban rail transit are often needed. With the advantages of flexibility, the feeder bus can fill the service gap left by existing public transit and reduce costs in specific scenarios [2]. Coordinated optimization of urban rail transit and feeder bus can further reduce traveling costs [3]. Nevertheless, the nonavailability of buses at origins and destinations is one of the main reasons why travelers give up public transit. In the background of the accelerated expansion of urban rail transit network, rational feeder routes for urban rail transit stations can strengthen the coordination of multilevel urban traffic modes and improve the sharing rate of public transportation [4] and operation efficiency [5].

Similar to feeder route design, bus route design problems have been researched intensively in the past decade. Since 
the transit system is a multimodal, multiproblem, and multispectral activity, researchers considered many concepts for determining the objectives such as service coverage, transfer coefficient, total traveling time, or total costs. However, there are conflicts between the above objectives. Different targets and constraints exist in every specific planning model. The objective functions of these problems are typically the total costs, including users' and operators' costs such as researches made by Lownes and Machemehl [6], Xiong et al. [7], Mohaymany and Gholami [8], Saeed and Kurauchi [9], and Yao et al. [10]. Mireia et al. [11] proposed a bilevel formulation to design bus routes of interurban services within a major city, where the objective function of the first level is intended to reduce costs of users and agency, and the performance of users was addressed in the second level. However, there are also some exceptions and many other factors are studied. For example, Chu [12] proposed an innovative mixed-integer programming model to design a bus route and timetable simultaneously. Based on fixedroute transit, Qiu et al. [13] identified a new category of transit policies, demi-flexible operating policies, where passenger costs function was defined as the performance measure. In addition to single-class public transit, Bagloee and Ceder [14] took categorization of stops, multiclass of transit vehicles, hierarchy planning, system capacity, and the integration between route design and frequency setting into consideration in the network design model. Lin and Wong [15] added the impact of the feeder bus on the service level of existing roads into the model. Other than constant traveling demand and road conditions, Yao et al. [16] formulated a robust optimization model for maximizing the efficiency of passenger trips. The model took into account the stochastic travel time by adding a buffer time to the average transit journey time due to uncertain traffic conditions to provide reliable transit service. Yan et al. [17] did similar research but introduced other constraints into the model, including the presence of overlapping bus lines and maximum allowable bus line directness. Chien et al. [18] presented a model to determine the optimal bus route location and its headway. The model considered intersection delays, irregular grid street patterns, heterogeneous demand distributions, and realistically geographic variations at the same time. From the perspective of the number of designed routes, Nikolic and Teodorović [19] proposed a bus network design model attempting to minimize the total number of transfers and the total travel time of all passengers and maximize the number of satisfied passengers without any constraint in the model. However, there was a limitation that the model took the shortest route between the origin and terminal as the final route. Shrivastava and O'Mahony [20] developed a model for the generation of the feeder route network and coordinated schedules at the same time.

The solution methods of problems can be categorized into four groups, namely, exact algorithm, heuristic, metaheuristic, and hybrid methods. Although heuristic and metaheuristic algorithms cannot get as accurate results as exact algorithms, they have been gaining more attention because of their higher computing performance in mediumscale and large-scale networks. In recent years, metaheuristic and hybrid algorithms have been widely used. Genetic algorithm [7, 21, 22], bee colony optimization [19], ant colony optimization [8], simulated annealing algorithm [17], and tabu search $[6,11,16]$ are usual metaheuristic methods to solve these problems. Based on the genetic algorithm, Yao et al. [10] utilized NSGA-II (nondominated sorting genetic algorithm II) to solve the multiobjective model. Nikolić and Teodorović [19] proposed an innovative BCO (bee colony optimization) algorithm consisting of two types of bees to optimize origins and terminals of bus routes through forward and backward passes. Bagloee and Ceder [14] encoded the search procedure through a genetic algorithm and equipped with an ant system to solve the complex problem. Hybrid methods, which combine the ability of different computational tools, hold many promises in terms of tackling complicated problems. Hu et al. [23] and Shrivastava and O'Mahony [24] developed hybrid methods to design route locations and timetables simultaneously. Cipriani et al. [25] proposed a hybrid method composed of a heuristic generation algorithm and a genetic algorithm. In another research, Yan et al. [17] subsequently implemented a heuristic solution approach based on the k-shortest path algorithm, simulated annealing algorithm, Monte Carlo simulation, and probit-type discrete choice model to solve the robust optimization model. Other algorithms were also applied to solve the problems. For instance, BPC (branchand-price-and-cut) [12] and TOPSIS (Technique for Order of Preference by Similarity to Ideal Solution) [15] were used to tackle similar problems. Kuan et al. [26] found that tabu search had better performance than simulated annealing, genetic algorithm, and ant colony optimization (ACO) for feeder network design problem in terms of the quality of the obtained solution regardless of the computation time needed.

Accessibility is vital for encouraging traffic mode shifts to reduce car reliance [27]. It is also found that incorporating accessibility as an explanatory variable can enhance the performance of active transport demand models [28] whereas accessibility via existing bus lines is ignored in some research studies when designing bus routes. Existing bus lines are inevitable in most areas. Ignoring them and ultimately designing new routes are likely to result in resource waste. To make the model more realistic, this paper took into account the impacts of existing bus lines. Moreover, many researchers have made efforts to optimize the bus network without taking into account urban rail stations and a single feeder bus route. Meanwhile, there usually is more than one urban rail station in a specific area of the city. Designing single feeder routes one by one may neglect the coordinated layout between routes. The problem of multiple feeder route design for multiple urban rail stations is further investigated in this study.

From the perspective of urban rail transit operators, a bus circulator system is a cost-effective means to connect urban rail stations to final destinations; therefore, supporting a more competitive multimodal transportation solution to increase the potential urban rail ridership may be a better choice [29]. Bus lines surrounding urban rail transit stations could not only meet transfer demand but also 
encourage transfer passengers who are traveling by walking, nonmotor vehicles, or taxis to move onto public transportation modes [30]. The circular route is adapted by the feeder bus in this paper. Accordingly, this paper develops a model to identify the existing transit service gap and optimizes multiple circular feeder bus routes. Feeder bus, which is also called minibus, shuttle bus, or community bus, refers to public transport vehicles with a relatively smaller size and providing short-distance feeder service for urban rail transit stations in this paper.

\section{Multiple Route Design Problem}

In this section, the problem description and several assumptions of this study are introduced. Then, the nonlinear mixed-integer model for the problem is proposed.

2.1. Problem Settings. Considering an area with existing bus lines, certain bus stops need to be provided for better feeder service because of their high feeder demand, long walking distance to urban rail stations, and a small number of existing bus lines. The connection between these bus stops and urban rail stations needs to be enhanced.

Consider a road network shown in Figure 1. The paper denotes this network by $G=(N, A)$, where $N$ is the set of nodes and $A$ is the set of links (street segments). Nodes, denoted by $i, j, k$, etc., which are typically intersections, existing bus stops, and urban rail stations, represent potential planned bus stops, where $i, j, k \in N$. A sequence of adjacent nodes defines any path used by transit passengers. A circular bus line can form a block service subarea $r$, where $N_{r}$ denotes the node set of $r$. Naturally, the whole network can also be denoted by $R$, where $r \in R$. $N_{r, c}$ denotes the set of candidate stop nodes composing a bus route in every subarea. We are assuming that the government or operators represent the number of newly planned bus routes $n_{r}$. Accordingly, there are also $n_{r}$ subareas. The paper studies a method to rationally partition the area, select candidate bus stops, and design bus routes to provide feeder service for urban rail stations.

This paper incorporates several modeling assumptions into the model construction to ease the complexity of the problem. The assumptions include the following: (a) based on the existing bus lines, the passenger demand assumed by the newly planned bus routes is equal to the ratio of the total demand between nodes to the number of existing bus lines plus one, and the demand is constant; (b) no varying traffic condition and no traffic accident are considered and any feeder bus runs at a known constant speed, so travel time on any link or route is fixed and merely proportional to travel distance; (c) headway of any feeder bus is constant and is equal to the ratio of total operating time to fleet size; (d) any feeder bus will provide sufficient capacity for its receiving passenger demand; and (e) passengers' arrival time

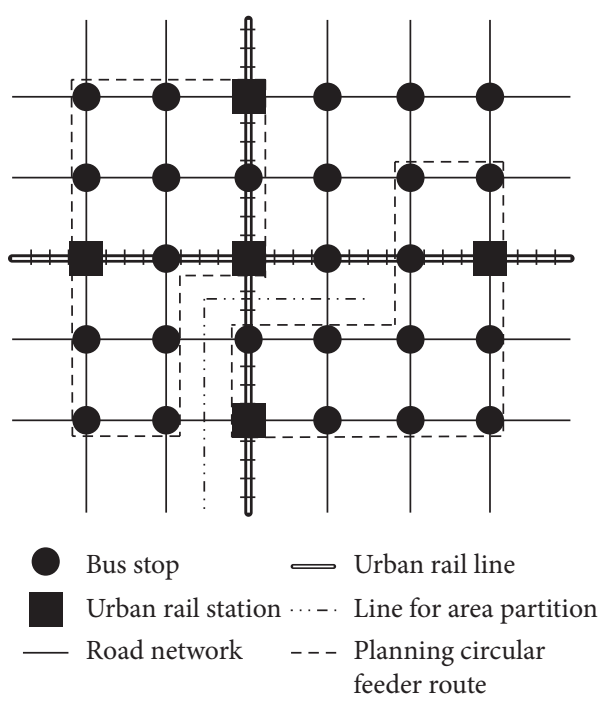

FIgURE 1: An example service network and planned feeder bus layout.

distributed evenly, which means the average waiting time is half of the headway.

2.2. Problem Formulation. Under the condition of utilizing existing bus stops and stations, operating costs are mainly relevant to fleet size, which principally depends on the number of newly planned bus routes and service frequency. This paper formulates a model to minimize passengers' traveling costs with a preset fleet size. Total costs in the whole area $\left(C_{t}\right)$ are calculated in the following way:

$$
C_{t}=\min \sum_{r \in R} C_{r}
$$

where $C_{r}$ denotes passengers' traveling costs in subarea $r$.

The problem is constructed as a mixed-integer program with a decision variable $z_{i}$, taking the value 1 if node $i$ is selected as a candidate stop and 0 otherwise. The second variable $w_{i j}$ is the integer number of passengers that travel from $i$ to $j$. Traveling costs in each subarea are a weighted sum of in-vehicle travel time, walking time, waiting time, and penalty of unserved passengers. Passengers would probably pay dollars to reduce travel time. The weights are the average monetary value of travel time (VOT) based on factors, such as the characteristics of passengers, the transportation mode, and the location. The traveling costs are formulated as follows:

$$
C_{r}=C_{r, \text { iv }}+C_{r, \text { pen }}+C_{r, w}+C_{r, w v}
$$

where $C_{r, i v}$ is passengers' in-vehicle travel costs in subarea $r$, $C_{r \text {,pen }}$ is penalty of unserved passengers in subarea $r, C_{r, w}$ is passengers' walking costs in subarea $r$, and $C_{r, w v}$ is passengers' waiting costs in subarea $r$. 
Formulation of every item of costs in a subarea is written as follows:

$$
\begin{aligned}
C_{r, \text { iv }} & =c_{\mathrm{iv}} \sum_{i \in N_{r, c}}\left(1+\frac{1}{c_{\text {feeder }, i}}\right)\left(\sum_{j \in N_{r, c} l\{i\}}\left(\frac{d_{i j}}{v_{i j}} \frac{w_{i j}}{1+n_{i, j, j \text { line }}}\right)+\delta_{i} \sum_{j \in N_{r, c} /\{i\}} w_{i j}\right), \\
C_{r, \text { pen }} & =c_{\text {pen }} \sum_{i \in N_{r, c}} \sum_{j \in N_{r} / N_{r, c}} w_{i j}, \\
C_{r, w} & =c_{w} \sum_{i \in N_{r, c}} \sum_{j \in N_{r, \text { rail }}}\left(w_{i j} \times d_{j, \text { rail }}\right), \\
C_{r, w v} & =c_{w v} \times \sum_{i \in N_{r, c}} \sum_{j \in N_{r, c} /\{i\}} \frac{w_{i j}}{1+n_{i, j, l i n e}} \times \sum_{i \in N_{r, c}}\left(\frac{\sum_{j=i+1} d_{i j} / v_{i j}+\delta_{i}+t_{\mathrm{acc}}+t_{\mathrm{dec}}}{n_{v}}\right), \\
\forall i & \in N_{r}, \quad \text { if } z_{i}=1, i \in N_{r, c},
\end{aligned}
$$

where $c_{\mathrm{iv}}$ is passenger's monetary in-vehicle costs per unit time, $c_{\text {pen }}$ is a passenger's monetary unserved penalty, $c_{w}$ is passenger's monetary walking costs per unit distance, $c_{w v}$ is passenger's monetary waiting costs per unit time, $w_{i j}$ is the number of passengers riding buses from stop $i$ to $j, c_{\text {feeder, } i}$ is feeder demand coefficient of bus stop $i, N_{r \text {,rail }}$ is a node set of stops connecting to urban rail stations in subarea $r, d_{j \text {,rail }}$ is the walking distance between node $j$ and the corresponding urban rail station, $d_{i j}$ is the distance between bus stop $i$ and $j$, $v_{i j}$ is the average operating speed of the feeder bus from node $i$ to $j, n_{i, j}$,line is the number of existing bus lines from bus stop $i$ to $j, n_{v}$ is the fleet size of a planned feeder bus route, $t_{\text {acc }}$ is accelerating time of a feeder bus for departure, $t_{\mathrm{dec}}$ is decelerating time of a feeder bus before dwelling, and $\delta_{i}$ is passenger's alight time at bus stop $i$.

This paper presented a feeder demand coefficient denoted by $c_{\text {feeder } i}$ to quantify feeder demand for each stop and identify targeted stops, formulated as

$$
\begin{aligned}
& c_{\text {feeder }, i}=d_{\mathrm{em}, i, \text { rail }}^{c_{\text {detour }}} \times\left(\frac{O_{i, \text { rail }}}{n_{i, \text { rail line }}}\right)^{1 / 2} \times \frac{d_{i k, \text { walking }}-d_{\text {max }, \text { rail }}}{d_{\text {max ,rail }}}, \\
& d_{\text {em }, i \text {,rail }}=\ln \left(2 \times O_{i, \text { rail }}+\sum_{j \in N_{i, \text { dbus }}} O_{j, \text { rail }} \times\left(1+\frac{d_{\text {max , bus }}-d_{i j, \text { walking }}}{d_{\text {max }, \text { bus }}}\right)\right),
\end{aligned}
$$

where $k$ is the nearest urban rail station to the bus stop $i$, $d_{\mathrm{em}, i \text {,rail }}$ is the processed demand riding buses from stop $i$ to urban rail stations, $c_{i \text {,detour }}$ is the detour coefficient from bus stop $i$ to urban rail station $k, O_{i \text {,rail }}$ is the number of passengers riding buses from stop $i$ to urban rail stations, $n_{i \text {,rail,line }}$ is the number of existing bus lines between bus stop $i$ and urban rail stations, $\left(d_{i j \text {,walking }} / d_{i k \text {,walking }}\right)$ is the shortest walking distance from stop $i$ to $j / k, N_{i, d \text { bus }}$ is node set within $d_{\text {max bus }}$ from node $i, d_{\max \text {,rail }}$ is the maximum attracting distance of a rail station, and $d_{\max \text {,bus }}$ is the maximum attracting distance of a bus stop.
The feeder demand coefficient of stops would be infinity if there are no existing bus lines within the range of $d_{\text {max } \text {,rail }}$ (e.g., $800 \mathrm{~m}$ in some papers [31,32]) of these stops to connect them to any urban rail station.

These stops are identified as necessarily responded stops that are provided with feeder service in any case. Integrating equations (3) and (8) into account, adding stops with high feeder demand coefficients into the model leads to low traveling costs, which means the model tends to select these nodes as candidate stops and fill the transit service gap efficiently.

Passenger alight time is calculated in the following way:

$$
\begin{array}{r}
\delta_{i}=4.0+1.7 \times s_{i}, \\
s_{i}=\sum_{j \in N_{r, c}\{\{i\}} w_{i j}, \quad \forall i \in N_{r, c},
\end{array}
$$

where $s_{i}$ is the number of passengers served by stop $i$.

The objective function is constrained by the following equation:

$$
\begin{aligned}
& \sum_{i \in N_{r} /\{j\}} x_{i j}=z_{i}, \quad \forall i \in N_{r}, \\
& \sum_{j \in N_{r} /\{i\}} x_{i j}=z_{j}, \quad \forall j \in N_{r},
\end{aligned}
$$

where $x_{i j}$ denotes a decision variable, taking the value 1 if bus travels from $i$ to $j$, and 0 otherwise.

Constraints of equations (11) and (12) are familiar TSP constraints that restrict the optimal solution to contain one incoming and one outgoing segment for every node in the route subset $N_{r, c}$ :

$$
\min \left(d_{i j}\right) \geq d_{m, \text { sta }}, \quad \forall i, j \in N_{r, c}, i \neq j .
$$


The constraint of equation (13) is the minimum stop spacing constraint, where $d_{m \text {,sta }}$ is the minimum allowed distance between bus stop $i$ and $j$ :

$$
\sum_{i \in N_{r, c}} \sum_{j=i+1} \frac{d_{i j}}{v_{i j}}+\delta_{i}+t_{\mathrm{acc}}+t_{\mathrm{dec}} \leq \mathrm{HE} \times n_{v} .
$$

The constraint of equation (14) is the maximum operating time of bus route $N_{r, c}$, aiming at guaranteeing reasonable service frequency at a given fleet size. $\mathrm{HE}$ is the maximum allowed headway.

\section{Solution Algorithm}

The fundamental problem of circular bus route design is planning a possible shortest bus route resembling the traveling salesman problem (TSP) in many respects. The bus needs to visit each candidate stop node in sequence and return to the origin node. Any candidate stop node is only allowed to be visited once. To solve this problem, the number of candidate stops, the exact candidate stops, and the operating route of each planned route have to be solved. Considering the high computational complexity of determining the above elements simultaneously for this problem, this paper presents a method to decompose the problem into three subproblems. In this paper, the three subproblems are sequential and designed to simplify the whole problem. Each subproblem is solved on the basis of the solution of the former subproblem. For example, candidate stops are selected in a reasonably divided area. And a bus route is designed based on the selected candidate stops. After a solution to the whole problem is found, the algorithm will go back to the first subproblem and try to find a better solution. The final solution is found through such iterations.

3.1. Problem Decomposition and Algorithm Selection. There are usually dozens of or hundreds of nodes in a planned area, and it is challenging to design multiple feeder routes directly because countless possible combinations of candidate stops exist. A circular bus route can surround a block service subarea under the circumstance that the government or operators preset the number of newly planned bus routes $n_{\mathrm{r}}$. Accordingly, the first step is to partition the entire area into $n_{\mathrm{r}}$ subareas to lay the foundation for designing a feeder route in each subarea.

Route design relies on candidate stop node set. With the limitation of operating costs, providing service for all nodes seems impossible. A certain number of candidate stop nodes need to be selected. The second step is to choose $n_{r, c}$ candidate stops from every subarea $r$. Moreover, as mentioned above, the set of candidate stop nodes is denoted by $N_{r, c}$.

After the above two procedures, the problem is simplified to an approximate traveling salesman problem. Namely, the last step is to find the shortest circular route to visit each candidate stop and determine the operating direction, by comparison, that is, operating in the same direction or two opposite directions.
Since network design is an NP-hard problem [33] and there is a combinatorial explosion as the number of nodes increases, metaheuristic algorithms are widely used methods to solve this kind of large-scale optimization problem. Tabu search uses a neighborhood search procedure to iteratively move from the current solution to the neighborhood until some stopping criterion has been satisfied. Based on the above problem decomposition, the second step is similar to the classic traveling salesman problem (TSP). Tabu search is a heuristic that, if used effectively, can promise an efficient near-optimal solution to the TSP $[34,35]$. The proposed methodology to solve the mixed-integer programming problem is based on the tabu search algorithm. Tabu search is a metaheuristic method in which the basic principle is to exploit neighborhoods of the current solution and improve optimization. Tabu search utilizes a neighborhood search procedure to iteratively move from the current solution to the neighborhood until some stopping criteria have been satisfied. To avoid falling into local optimum, certain moves are forbidden, for example, tabu move. The solution list, including tabu moves, is called tabu list. Tabu moves in the tabu list are released after a set time named tabu tenure. Aspiration criterion in tabu search permits certain moves in the tabu list to overcome any tabu status. Detailed procedures of the proposed algorithm are shown in Figure 2.

3.2. Feeder Area Partition. The principle of feeder area partition optimization is to move specific boundary nodes into an adjacent subarea to search for a better solution by tabu search.

3.2.1. Initial Solution. Taking the geographic coordinates of all nodes as input variables, the K-means clustering algorithm, an unsupervised learning method, is used to divide nodes into $n_{r}$ sets. Nodes in the same set compose a subarea. After calculating the coordinate of a subarea's center point, the urban rail station node nearest to the center point of the subarea is divided into the subarea as a feeder urban rail station. The initial solution of area partition (the node set of each subarea) is determined.

3.2.2. Neighborhood Generation. Define that the searchable neighborhoods are the boundary nodes that link to adjacent subareas. The subarea with the highest traveling costs is selected as the moving-out subarea, and the adjacent area with the lowest traveling cost is the moving-in subarea. No more than two coterminous nodes are randomly selected to move. A move is feasible if it satisfies the aspiration criterion or does not exist in the tabu list. Then the reverse move is marked as a tabu. Likewise, a move is infeasible if it exists in the tabu list and does not satisfy the aspiration criterion, under which circumstance new nodes need to be selected.

3.2.3. Evaluation and Updating. After completing the move, the total traveling costs of the entire area are calculated and compared with the incumbent solution to evaluate and update the solution. For this feeder area partition problem, this paper 


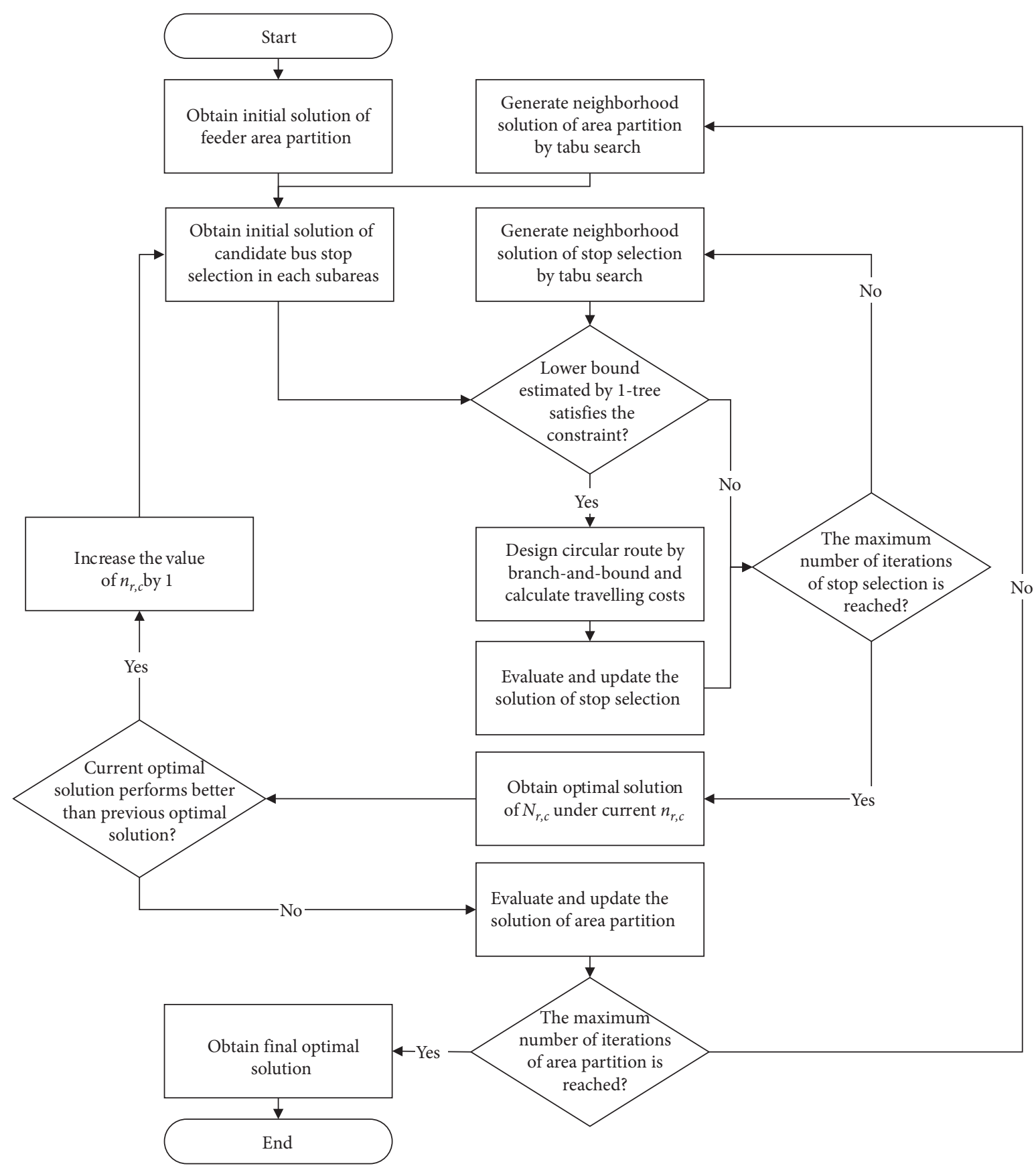

FIGURE 2: Flowchart of the proposed algorithm.

suggests a constant tenure and two tabu search rules: (a) if a neighborhood solution reached by a move performs better than the current optimal solution, update the incumbent solution and current optimal solution with the neighborhood solution and mark the reverse move as a tabu; (b) if a neighborhood solution reached by a move performs better than the incumbent solution or the move is not a tabu, update the incumbent solution and mark the reverse move as a tabu.

\subsection{Candidate Stop Selection and Route Design in a Subarea.} The principle of candidate stop selection optimization is to generate neighborhood solutions of node selection by tabu search. The route design problem is solved by a branch-andbound solver.

3.3.1. Initial Solution. The lower bound of feeder bus stop spacing is around $300 \mathrm{~m}$ [36-38]. Considering the regular shortest length of a feeder bus route, the minimum number of feeder bus stops for a circular route is 6 , that is, the minimum $n_{r, c}$ is 6 . If $n_{r, c}$ is equal to 6 , select stops with the most significant feeder demand coefficient as the initial solution. If $n_{r, c}$ is more significant than 6 , select stops of the previous optimal solution and one stop out of 
the previous optimal solution but with the most significant feeder demand coefficient as the initial solution.

3.3.2. Neighborhood Generation. Define that the searchable neighborhood is bus stop nodes out of the incumbent solution, and feeder urban rail stations are forbidden to move. The paper employs a method of selecting a bus stop to leave the incumbent solution and several options to fill the emptiness in the candidate bus stops set $N_{r, c}$.

The node $i$ leaving the solution is selected randomly, provided it is not a feeder urban rail station and not tabu. In order to exploit neighborhoods and improve optimization efficiency, move score $p_{i j}$ is defined, aiming at measuring the potential performance of a move. To equalize the magnitude and variability of all possible moves, variable standardization is conducted. In addition, a random disturbance is introduced into the formulation to avoid entirely relying on calculation and falling into local optimum. The moving score is formulated as follows:

$$
\begin{aligned}
p_{i j} & =p_{i j, \text { sta }}+\delta, \quad-1 \leq \delta \leq 1, \\
p_{i j, \text { sta }} & =\frac{p_{i j, \text { raw }}-\mu}{\sigma}, \\
p_{i j, \text { raw }} & =\sum_{j \in N_{r, c} l\{i\}} \frac{w_{i j}}{d_{i j}}, \quad \forall j \in \frac{N_{r}}{N_{r, c}},
\end{aligned}
$$

where $p_{i j}$ is the move score if select node $i$ leaving the solution and $j$ entering the solution, $p_{i j \text {,sta }}$ is the standardized move score, $p_{i j \text {,raw }}$ is the raw move score without standardization, $\delta$ is a random disturbance between -1 and $1, \mu$ is the mean value of all possible raw move scores, and $\sigma$ is the standard variance of all possible raw move scores.

This attribute is roughly a ratio of the demand served by the candidate stop to the total distance from that stop to the remainder of the set $N_{r, c}$. When exploiting neighborhood, the method tends to select node $j$ with the highest value of $p_{i j}$ to replace node $i$. That is to say, the algorithm is attempting to incorporate bus stops that serve a high amount of demand and are near to the other candidate stops in the set $N_{r, c}$. As a result, the algorithm tends to favor clusters of stops. The move has to satisfy specific rules about tabu and aspiration criterion similar to neighborhood generation part in the feeder area partition chapter, or it has to be reselected.

3.3.3. Evaluation and Updating. Once a neighborhood generation is completed and candidate bus stops are determined, the remaining circular route design problem is a traveling salesman problem which the solution methods have been made great efforts on. The exact method branchand-bound is called to solve the shortest circular route in each subarea, and the best operating direction is obtained by calculating and comparing traveling costs in different directions. Final total traveling costs are gained and the quality of the neighborhood solution is evaluated.

Tabu tenure indicates the size of the tabu list and has an essential effect on the algorithm's performance in avoiding falling into local optimum. Constant tabu tenure is not affected by whether the solution is feasible or enhanced. To adapt to the process of solving, an adaptive tabu tenure is suggested [27], which can increase the value of tabu tenure when the current optimal solution gets updated or decrease the value when the incumbent solution is not updated after several moves. Adaptive tabu search rules are suggested to adjust tabu tenure along with the search process in terms of the quality of recently found solutions:

(a) If a neighborhood solution reached by a move performs better than the current optimal solution, update the incumbent solution and current optimal solution with the neighborhood solution, mark the reverse move as a tabu, and increase the value of tabu tenure by 1

(b) If a neighborhood solution reached by a move performs better than the incumbent solution or the move is not a tabu, update the incumbent solution with the neighborhood solution, mark the reverse move as a tabu, and remain the value of tabu tenure

(c) If the current optimal solution is not updated after certain moves, decrease the value of tabu tenure by 1

After the current optimal solution is obtained under the current $n_{r, c}$ value, increase the $n_{r, c}$ value by 1 and gain the new solution until the optimal global solution including $N_{r, c}$ and $n_{r, c}$ is obtained.

\subsubsection{Computing Efficiency Enhancement}

(1) Lower Bound of Operating Time Estimation. If candidate stop nodes are scattered, they are far from each other, causing a long operating time exceeding the maximum operating time constraint of equation (14). Under this circumstance, an infeasible solution is generated and the subsequent route design solution results in unnecessary computation. Understandably, the solution is infeasible and no more computation is necessary if the lower bound of operating time does not satisfy the constraint. The paper proposes a method of estimating the lower bound of operating time to identify and eliminate infeasible solutions prior to evaluation.

As mentioned above, the circular route design problem can be considered as a traveling salesman problem. A method of establishing a lower bound for the TSP is the 1tree, which is a tree that consists of a vertex attached with two edges to a spanning tree. A minimum spanning tree can provide both a rough lower and upper bound for the TSP. Further improvement of the lower bound can be obtained through 1-tree as it has an extra arc that creates a cycle. The largest result of all found 1-tree is a tighter lower bound than the others. 1-tree solution can be found in polynomial time, while the branch-and-bound solution cannot always be found in polynomial time. As a result, the preprocessing steps to find a 1-tree solution will enhance computing efficiency. 


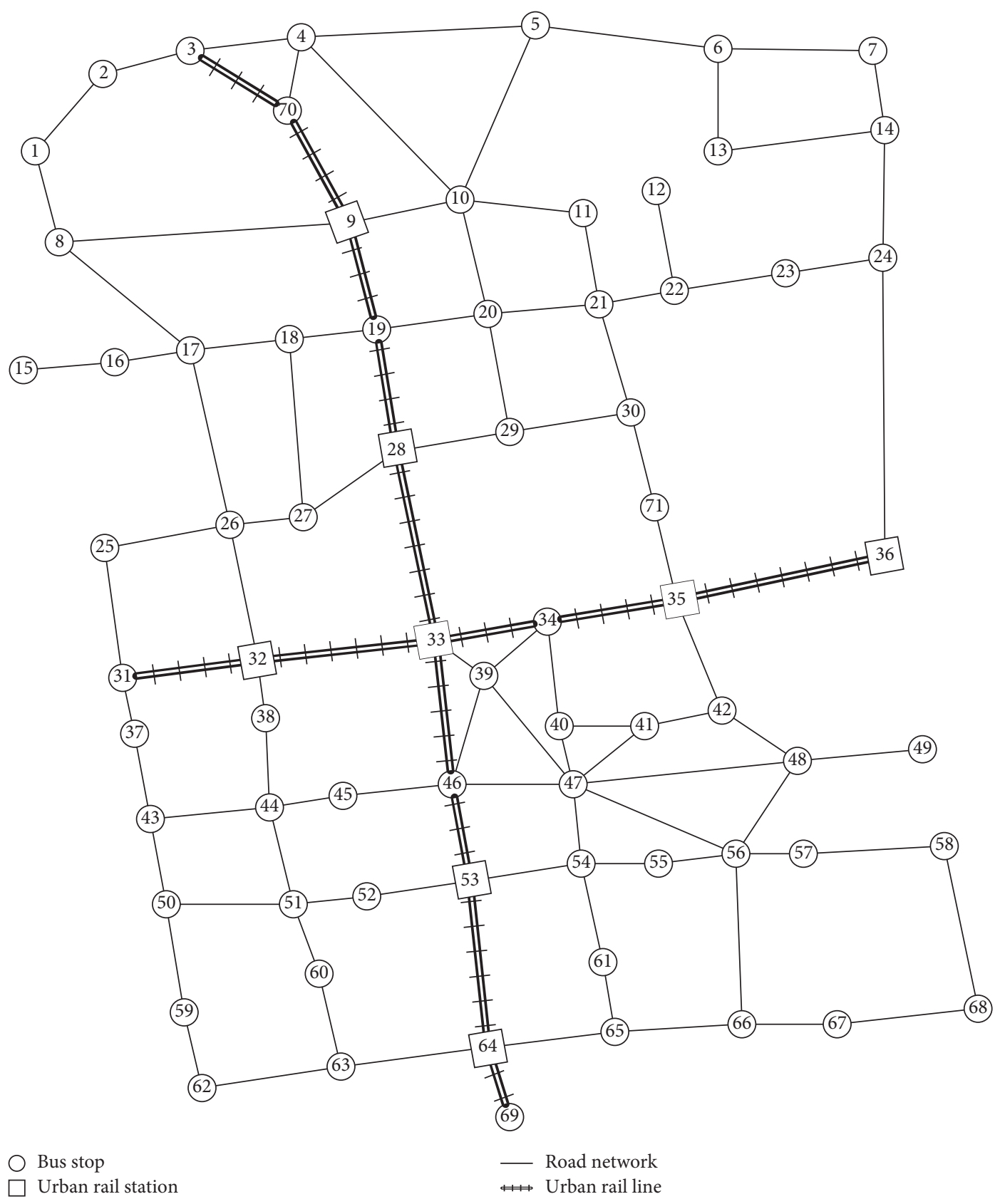

FIgURE 3: Topological road network of the applied area.

(2) Optimization Stopping Criterion. The proposed candidate stop selection method needs to determine the number of candidate stops (that is, the value of $n_{r, c}$ ) in advance. Solutions under the different values of $n_{r, c}$ need to be obtained. Increasing the number of candidate stops leads to both sides' effects. On the one hand, the service area is expanded and more passengers are served, so the unserved penalty decreases. On the other hand, service frequency decreases and operating time increases under a fixed fleet size as a result of additional route length and dwelling time. Passengers will spend more time waiting and riding. Nodes in the current optimal solution are regarded as stops with more positive benefits than unselected nodes. Potential benefits of unselected nodes may be negative because the additional waiting
TABLE 1: Value of related parameters in the model.

\begin{tabular}{lc}
\hline Parameter & Value \\
\hline$c_{\text {iv }}$ & 0.6 (yuan $/$ min) \\
$c_{\text {pen }}$ & 4.0 (yuan $/$ passenger) \\
$c_{w}$ & 0.015 (yuan $/$ meter) \\
$c_{w v}$ & 0.8 (yuan $/ \mathrm{min})$ \\
$v_{i j}$ & $20(\mathrm{~km} / \mathrm{h})$ \\
$d_{\max , \text { rail }}$ & $800($ meter $)$ \\
$t_{i, \text { acc }}$ & $6($ second) \\
$t_{i, \text { dec }}$ & $4($ second) \\
$d_{m, \text { sta }}$ & 300 (meter) \\
HE & 12 (min) \\
$n_{v}$ & 2 \\
$d_{\max , \text { bus }}$ & 400 (meter) \\
\hline
\end{tabular}




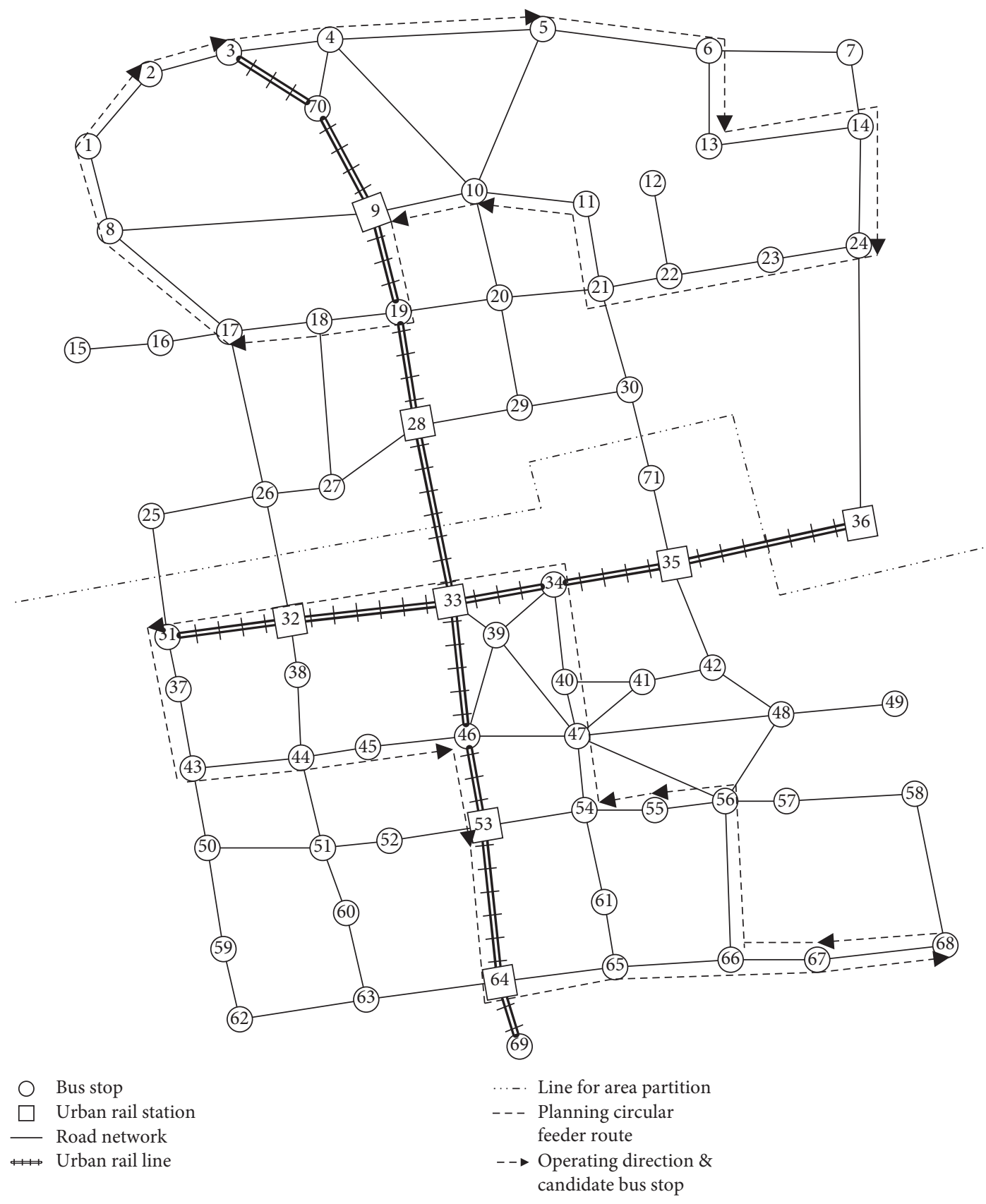

FIgURE 4: Optimum circular feeder bus routes.

time costs and riding time costs outweigh the reduction of the unserved demand penalty. Accordingly, if the marginal benefits of adding a candidate stop are negative, the current optimal solution is considered as an optimal global solution and candidate stop optimization stops. There is no need to enumerate all possible values of $n_{r, c}$ and thereby unnecessary computation is avoided.

\section{Application and Performance}

To check the validity of the proposed algorithm, a case study in a downtown area of Suzhou, China, is conducted. The quality of solutions and computing efficiency is verified by comparison.
4.1. Computation Results. By applying the proposed method to a practical case, the validity and practicability of it are examined. The application area is the downtown area inside the Waicheng River of Suzhou city, a major city located in Jiangsu Province of China. It covers around 14.2 square kilometers. Before application, there is a need to simplify the actual road network topology into a simple road network composed of several nodes and links, as shown in Figure 3, where nodes are normally intersections, existing bus stops, and urban rail stations, and links are street segments.

OD demand between every two nodes is acquired through a transit corporation. According to the income information in Suzhou Statistical Yearbook, monetary cost 


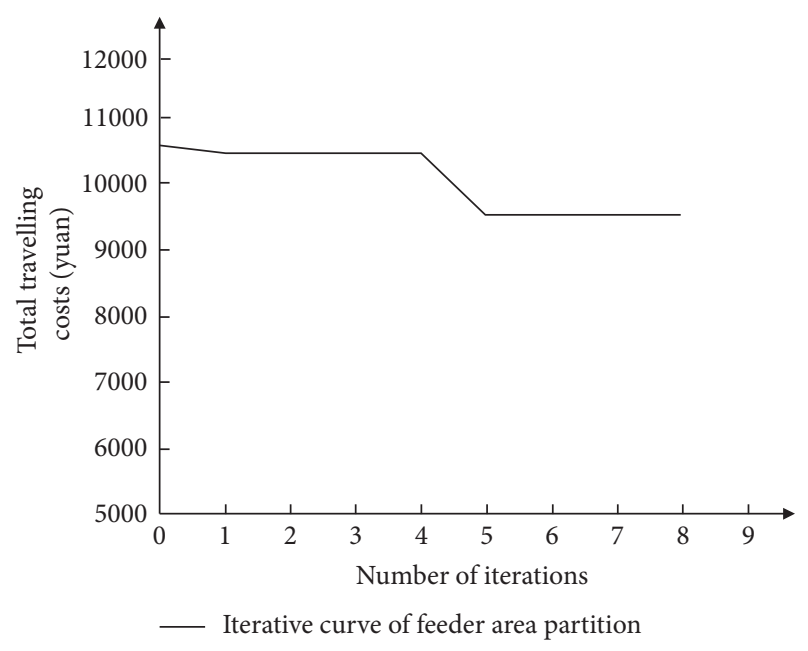

FIGURE 5: Iterative curve of the feeder area partition.

parameters in the model are estimated and given in Table 1. Preset that the number of newly planned feeder bus routes is 2 , and the fleet size of a route is 2 , meaning that maximum operating time is 24 minutes for a circular route. The solutions of two new planned circular feeder bus routes are shown in Figure 4. As shown in Figure 4, new planned bus stops are mainly nodes at the edge of the areas. According to the data, these nodes are in lack of enough bus services to accommodate high passenger volumes or are even not served by any bus lines. As a result, the paper thinks the provided feeder bus service is reasonable and can fill the service gap efficiently.

4.2. Computing Efficiency and Solution Quality. The validity of the proposed feeder area partition algorithm is checked, and the iterative curve is shown in Figure 5. Total traveling costs of the optimal solution through nine iterations of feeder area partition optimization are reduced by $9.63 \%$ compared to the initial solution. The method can indeed reduce total traveling costs while requiring nine times of computation time. The optimal solution is similar to the first solution obtained by spatial clustering. The method reduces traveling costs to some extent but it is more time consuming, especially in the larger-scale road network. Therefore, it is considered that the multiple circular feeder bus route design problem can be simplified to a single route design problem by taking the initial solution of area partition obtained by spatial clustering as the optimal solution to enhance computing efficiency in a large-scale network.

To check the validity of the proposed method of candidate stops selection, the second part of the entire algorithm, a circular feeder bus route is designed in a subarea with 25 nodes, including one feeder urban rail station and two necessarily responded stops. Enumerate 7,315 possible candidate stops combinations containing seven stops and total traveling costs for each combination are calculated, through which the optimal global solution is obtained. Setting the maximum number of iterations to 70, two optimal solutions are obtained by the proposed tabu search

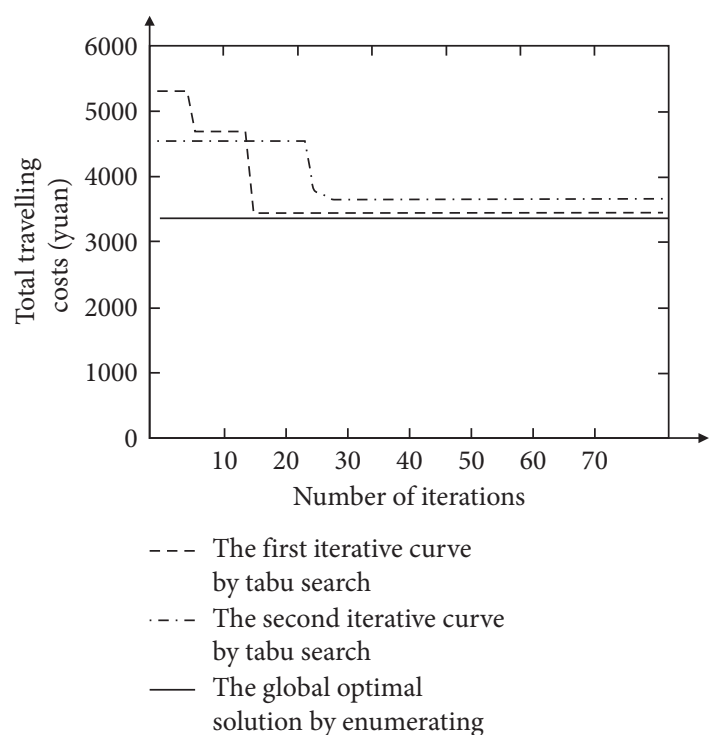

FIGURE 6: Iterative curves of two optimal solutions' total traveling costs.

algorithm after two repetitive calculations. Iterative curves of traveling costs of two optimal solutions are obtained and shown in Figure 6. Optimal solutions found by the tabu algorithm probably are not the optimal global solution, while the gap between them is, respectively, $8.87 \%$ and $2.77 \%$, typically smaller than $10 \%$. The value of the objective function decreases rapidly at the start and begins to get stabilized after around the 30th iteration. In conclusion, the paper thinks the proposed tabu search algorithm can gain a high-quality optimal solution while dramatically reducing time consumption.

\section{Conclusions}

This paper proposed a nonlinear mixed-integer programming model addressing multiple circular feeder bus route design problems. To take into account the existing bus lines of an area, the paper defined a feeder demand coefficient to quantify feeder demand for each bus stop and identify the service gap. As a result, the operators can provide feeder service for targeted stops. The feeder demand coefficient was introduced into the model. The proposed model aimed at minimizing the total traveling costs of passengers and tended to select nodes with a high value of feeder demand coefficient.

Since network design is a large combinational optimization problem whose global optimal solution is challenging to find, a method based on the tabu search algorithm was presented to solve the problem. Lower bound of TSP estimated by 1-tree and optimization stopping criterion were suggested to enhance computing efficiency. Moreover, a method was proposed to decompose the problem into three subproblems, including feeder area partition, candidate stops selection, and route design to reduce complexity. Based on the results of the application, the decomposition of the problem was valid and the algorithm was demonstrated to gain a high-quality optimal solution while dramatically 
reducing time consumption. The proposed method could effectively identify and fill the service gap and strengthen the coordination of multilevel public traffic modes. At last, through a comparison of optimal solutions, the paper provided a possibility of simplifying a multiple circular feeder route design problem to a single circular route design problem by spatial clustering.

In conclusion, the paper made two contributions to the feeder bus route design problem. An innovative model to fill the service gap was established and an effective algorithm was suggested. Moreover, the possibility of simplifying the problem was provided.

In areas with a mature public transport system, the service gap is sparse. Designing new feeder routes probably leads to long operating distance and stop spacing (at most six blocks in this paper's application) to visit each service gap. Under such circumstances, combining adjusting existing bus routes and designing new feeder routes together is probably a more cost-effective way. The paper made primary efforts on approaches to tackling route design problems. The maximum headway of the feeder bus is constrained in the model, so the specific schedule and the optimal headway are not solved. An advanced schedule in the intermodal system can reduce passengers' waiting time and improve satisfaction. So coordinated schedule optimization of the feeder bus by utilizing existing schedules of urban rail transit needs to be studied.

Furthermore, the paper only focused on single-mode transit route design. Coordinated optimization with other hierarchies of bus routes and multimode feeder systems providing various services for urban rail transit stations is essential to be considered. Public transportation systems have diverse characteristics and rules in different countries. Also, passengers have different traveling preferences and demands, depending on their various habits and the background of family and region. Stochasticity of the traveling demand and road conditions also affects the reliability and performance of the feeder bus. To improve their level of satisfaction, specific objectives and factors are needed.

\section{Data Availability}

The data used to support the findings of this study are available from the corresponding author upon request.

\section{Conflicts of Interest}

The authors declare that there are no conflicts of interest regarding the publication of this paper.

\section{Acknowledgments}

This research was supported by Scientific Research Foundation for Advanced Talents of Nanjing Forestry University (Grant no. 163106041), Project of Postgraduate Research \& Practice Innovation Program of Jiangsu Province (Grant nos. KYLX16-0276), China Scholarship Council (Grant no. 201806090209), and the Project of Philosophy and Social
Science Research in Jiangsu University (Grant no. 2020SJA0125). The authors also thank Zhenjun Zhu (College of Automobile and Traffic Engineering, Nanjing Forestry University) for his valuable comments to perfect the paper and thank Suzhou Planning and Design Institute Co., Ltd., for data support.

\section{References}

[1] D. Birago, S. Opoku Mensah, and S. Sharma, "Level of service delivery of public transport and mode choice in Accra, Ghana," Transportation Research Part F: Traffic Psychology and Behaviour, vol. 46, pp. 284-300, 2017.

[2] A. Gholami and A. S. Mohaymany, "Economic conditions for minibus usage in a multimodal feeder network," Transportation Planning and Technology, vol. 34, no. 8, pp. 839-856, 2011.

[3] M. Almasi, A. Sadollah, S. Kang, and M. Karim, "Optimization of an improved intermodal transit model equipped with feeder bus and railway systems using metaheuristics approaches," Sustainability, vol. 8, no. 537, pp. 1-27, 2016.

[4] S. C. Wirasinghe, L. Kattan, M. M. Rahman, J. Hubbell, R. Thilakaratne, and S. Anowar, "Bus rapid transit-a review," International Journal of Urban Sciences, vol. 17, no. 1, pp. 1-31, 2013.

[5] L. Deng, W. Gao, Y. Fu, and W. Zhou, "Optimal design of the feeder-bus network based on the transfer system," Discrete Dynamics in Nature and Society, vol. 2013, Article ID 483682, 10 pages, 2013.

[6] N. E. Lownes and R. B. Machemehl, "Exact and heuristic methods for public transit circulator design," Transportation Research Part B: Methodological, vol. 44, no. 2, pp. 309-318, 2010.

[7] J. Xiong, W. Guan, L. Song, A. Huang, and C. Shao, "Optimal routing design of a community shuttle for metro stations," Journal of Transportation Engineering, vol. 139, no. 12, pp. 1211-1223, 2013.

[8] A. S. Mohaymany and A. Gholami, "Multimodal feeder network design problem: ant colony optimization approach," Journal of Transportation Engineering, vol. 136, no. 4, pp. 323-331, 2010.

[9] K. Saeed and F. Kurauchi, "Design of multimodal transit services with stochastic demand," International Journal of Transportation, vol. 5, no. 3, pp. 1-16, 2017.

[10] B. Yao, Q. Cao, L. Jin, M. Zhang, and Y. Zhao, "Circle line optimization of shuttle bus in central business district without transit hub," PROMET-TrafficeTransportation, vol. 29, no. 1, pp. 45-55, 2017.

[11] R. R. Mireia, E. Miquel, and T. César, "The design of interurban bus networks in city centers," Transportation Research Part A: Policy and Practice, vol. 46, no. 8, pp. 1153-1165, 2012.

[12] J. C. Chu, "Mixed-integer programming model and branchand-price-and-cut algorithm for urban bus network design and timetabling," Transportation Research Part B: Methodological, vol. 108, pp. 188-216, 2018.

[13] F. Qiu, J. Shen, X. Zhang, and C. An, "Demi-flexible operating policies to promote the performance of public transit in lowdemand areas," Transportation Research Part A: Policy and Practice, vol. 80, pp. 215-230, 2015.

[14] S. A. Bagloee and A. A. Ceder, "Transit-network design methodology for actual-size road networks," Transportation Research Part B: Methodological, vol. 45, no. 10, pp. 17871804, 2011. 
[15] J.-J. Lin and H.-I. Wong, "Optimization of a feeder-bus route design by using a multiobjective programming approach," Transportation Planning and Technology, vol. 37, no. 5, pp. 430-449, 2014.

[16] B. Yao, P. Hu, X. Lu, J. Gao, and M. Zhang, “Transit network design based on travel time reliability," Transportation Research Part C: Emerging Technologies, vol. 43, pp. 233-248, 2014.

[17] Y. Yan, Z. Liu, Q. Meng, and Y. Jiang, "Robust optimization model of bus transit network design with stochastic travel time," Journal of Transportation Engineering, vol. 139, no. 6, pp. 625-634, 2013.

[18] S. Chien, Z. Yang, and E. Hou, "Genetic algorithm approach for transit route planning and design," Journal of Transportation Engineering, vol. 127, no. 3, pp. 200-207, 2001.

[19] M. Nikolić and D. Teodorović, "Transit network design by bee colony optimization," Expert Systems with Applications, vol. 40, no. 15, pp. 5945-5955, 2013.

[20] P. Shrivastava and M. O’Mahony, “A model for development of optimized feeder routes and coordinated schedules-a genetic algorithms approach," Transport Policy, vol. 13, no. 5, pp. 413-425, 2006.

[21] Z. Zhu, X. Guo, J. Zeng, and S. Zhang, "Route design model of feeder bus service for urban rail transit stations," Mathematical Problems in Engineering, vol. 2017, Article ID 1090457, 6 pages, 2017.

[22] S. L. Dhingra and A. Verma, "Feeder bus routes generation within integrated mass transit planning framework," Journal of Transportation Engineering, vol. 131, no. 11, pp. 822-834, 2005.

[23] Y. Hu, Q. Zhang, and W. Wang, "A model layout region optimization for feeder buses of rail transit," Procedia-Social and Behavioral Sciences, vol. 43, pp. 773-780, 2012.

[24] P. Shrivastava and M. O’Mahony, "Use of a hybrid algorithm for modeling coordinated feeder bus route network at suburban railway station," Journal of Transportation Engineering, vol. 135, no. 1, pp. 1-8, 2009.

[25] E. Cipriani, S. Gori, and M. Petrelli, "Transit network design: a procedure and an application to a large urban area," Transportation Research Part C: Emerging Technologies, vol. 20, no. 1, pp. 3-14, 2012.

[26] S. N. Kuan, H. L. Ong, and K. M. Ng, "Solving the feeder bus network design problem by genetic algorithms and ant colony optimization," Advances in Engineering Software, vol. 37, no. 6, pp. 351-359, 2006.

[27] S. Mavoa, K. Witten, T. McCreanor, and D. O'Sullivan, "GIS based destination accessibility via public transit and walking in Auckland, New Zealand," Journal of Transport Geography, vol. 20, no. 1, pp. 15-22, 2012.

[28] T. Saghapour, S. Moridpour, and R. G. Thompson, "Enhancing active transport demand modelling by incorporating accessibility measures," Cities, vol. 78, pp. 206-215, 2018.

[29] Y. Yu, R. B. Machemehl, and C. Xie, "Demand-responsive transit circulator service network design," Transportation Research Part E: Logistics and Transportation Review, vol. 76, pp. 160-175, 2015.

[30] J. Wu, Y. Zheng, and X. Chen, "Approaches to planning of subway station transfer facility in urban areas," Journal of Tongji University (Natural Science), vol. 36, no. 11, pp. 15011506, 2008.

[31] R. Daniels and C. Mulley, "Explaining walking distance to public transport: the dominance of public transport supply," Journal of Transport and Land Use, vol. 6, no. 2, pp. 5-20, 2013.
[32] T. M. Rahul and A. Verma, "A study of acceptable trip distances using walking and cycling in Bangalore," Journal of Transport Geography, vol. 38, pp. 106-113, 2014.

[33] Z. Yang, B. Yu, and C. Cheng, "A parallel ant colony algorithm for bus network optimization," Computer-Aided Civil and Infrastructure Engineering, vol. 22, no. 1, pp. 44-55, 2007.

[34] W. B. Carlton and J. W. Barnes, "Solving the travelingsalesman problem with time windows using tabu search," IIE Transactions, vol. 28, no. 8, pp. 617-629, 1996.

[35] M. Malek, M. Guruswamy, M. Pandya, and H. Owens, "Serial and parallel simulated annealing and tabu search algorithms for the traveling salesman problem," Annals of Operations Research, vol. 21, no. 1, pp. 59-84, 1989.

[36] Z. Zhu, X. Guo, H. Chen, J. Zeng, and J. Wu, "Optimization of urban mini-bus stop spacing: a case study of Shanghai (China)," Tehnicki Vjesnik-Technical Gazette, vol. 24, no. 3, pp. 949-955, 2017.

[37] A. Tirachini, "The economics and engineering of bus stops: spacing, design and congestion," Transportation Research Part A: Policy and Practice, vol. 59, pp. 37-57, 2014.

[38] Á. Ibeas, L. dell'Olio, B. Alonso, and O. Sainz, "Optimizing bus stop spacing in urban areas," Transportation Research Part E: Logistics and Transportation Review, vol. 46, no. 3, pp. $446-458,2010$. 